\title{
The FETI Based Domain Decomposition Method for Solving 3D-Multibody Contact Problems with Coulomb Friction *
}

\author{
Radek Kučera ${ }^{1}$, Jaroslav Haslinger $^{2}$, and Zdeněk Dostál ${ }^{3}$ \\ 1 VŠB-TU Ostrava, Department of Mathematics, radek.kucera@vsb.cz \\ 2 Charles University, Department of Metal Physics, haslin@met.mff.cuni.cz \\ 3 VŠB-Technical University Ostrava, Department of Applied Mathematics, \\ zdenek.dostal@vsb.cz
}

Summary. The contribution deals with the numerical solving of contact problems with Coulomb friction for 3D bodies. A variant of the FETI based domain decomposition method is used. Numerical experiments illustrate the efficiency of our algorithm.

\section{Introduction}

The FETI method was proposed by Farhat and Roux [1992] for parallel solution of problems described by elliptic partial differential equations. The key idea is elimination of the primal variables so that the original problem is reduced to a small, relatively well conditioned quadratic programming problem in terms of the Lagrange multipliers. Then the iterative solver is used to compute the solution.

Our recent papers (see Dostál et al. [2002] or Haslinger et al. [2002]) apply the FETI procedure to the contact problems with Coulomb friction in $2 \mathrm{D}$. It leads to the sequence of quadratic programming problems with simple inequality bounds so that the fast algorithm based on an active set strategy and an adaptive precision control (see Dostál and Schöberl [2003]) can be used directly. The situation is not so easy in $3 \mathrm{D}$. The reason is that the tangential contact stress has two components in each contact node which are subject to quadratic inequality constraints. Fortunately the structure of this constraints is relatively simple: the vector whose components are the tangential contact stresses belongs to a circle in $\mathbb{R}^{2}$ with the center at the origin and a given radius. A convenient piecewise linear approximation of the circle can be defined by the intersection of squares rotated of a constant angle $\alpha$. Doing this approximation at all the contact nodes, we obtain a new quadratic

* Supported by grant GAČR 101/01/0538, MSM ME641 and MSM 272400019. 
programming problem with bound and equality constraints that can be efficiently solved by the algorithm based on the augmented Lagrangian (see Dostál et al. [2003]). The implementation details for the problem with one body on the rigid foundation can be found in Haslinger et al. [2003]. Here, we shall extend our method to 3D-multibody problems and show how to reduce the size of the solved quadratic programming problem by means of the mixed finite element method.

\section{Formulation of the problems}

Let us consider a system of elastic bodies that occupy in the reference configuration bounded domains $\Omega^{p} \subset \mathbb{R}^{3}, p=1,2, \ldots, s$, with sufficiently smooth boundaries $\Gamma^{p}$ that are split into three disjoint parts $\Gamma_{u}^{p}, \Gamma_{t}^{p}$ and $\Gamma_{c}^{p}$ so that $\Gamma^{p}=\overline{\Gamma_{u}^{p}} \cup \overline{\Gamma_{t}^{p}} \cup \overline{\Gamma_{c}^{p}}$. Let us suppose that the zero displacements are prescribed on $\Gamma_{u}^{p}$ and that the surface tractions of density $\mathbf{t}^{p} \in\left(L^{2}\left(\Gamma_{t}^{p}\right)\right)^{3}$ act on $\Gamma_{t}^{p}$. Along $\Gamma_{c}^{p}$ the body $\Omega^{p}$ may get into unilateral contact with some other of the bodies. Finally we suppose that the bodies $\Omega^{p}$ are subject to the volume forces of density $\mathbf{f}^{p} \in\left(L^{2}\left(\Omega^{p}\right)\right)^{3}$.

To describe non-penetration of the bodies, we shall use linearized nonpenetration condition that is defined by a mapping $\chi: \Gamma_{c} \longrightarrow \Gamma_{c}, \Gamma_{c}=$ $\bigcup_{p=1}^{s} \Gamma_{c}^{p}$, which assigns to each $\mathbf{x} \in \Gamma_{c}^{p}$ some nearby point $\chi(\mathbf{x}) \in \Gamma_{c}^{q}, p \neq q$. Let $\mathbf{v}^{p}(\mathbf{x}), \mathbf{v}^{q}(\boldsymbol{\chi}(\mathbf{x}))$ denote the displacement vectors at $\mathbf{x}, \boldsymbol{\chi}(\mathbf{x})$, respectively. Assuming the small displacements, the non-penetration condition reads

$$
v_{n}^{p}(\mathbf{x}) \equiv\left(\mathbf{v}^{p}(\mathbf{x})-\mathbf{v}^{q}(\boldsymbol{\chi}(\mathbf{x}))\right) \cdot \mathbf{n}^{p}(\mathbf{x}) \leq \delta^{p}(\mathbf{x}),
$$

where $\delta^{p}(\mathbf{x})=(\boldsymbol{\chi}(\mathbf{x})-\mathbf{x}) \cdot \mathbf{n}^{p}(\mathbf{x})$ is the initial gap and $\mathbf{n}^{p}(\mathbf{x})$ is the critical direction defined by $\mathbf{n}^{p}(\mathbf{x})=(\boldsymbol{\chi}(\mathbf{x})-\mathbf{x}) /\|\boldsymbol{\chi}(\mathbf{x})-\mathbf{x}\|$ or, if $\boldsymbol{\chi}(\mathbf{x})=\mathbf{x}$, by the outer unit normal vector to $\Gamma_{c}^{p}$.

We start with the weak formulation of an auxiliary problem, called the contact problem with given friction. To this end we introduce the space of virtual displacements

$$
V=\left\{\mathbf{v}=\left(\mathbf{v}^{1}, \ldots, \mathbf{v}^{s}\right) \in \prod_{p=1}^{s}\left(H^{1}\left(\Omega^{p}\right)\right)^{3}: \mathbf{v}^{p}=0 \text { on } \Gamma_{u}^{p}\right\}
$$

and its closed convex subset of kinematically admissible displacements

$$
\mathcal{K}=\left\{\mathbf{v} \in V: v_{n}^{p}(\mathbf{x}) \leq \delta^{p}(\mathbf{x}) \text { for } \mathbf{x} \in \Gamma_{c}^{p}\right\},
$$

where $\mathbf{n}^{p} \in\left(L^{\infty}\left(\Gamma_{c}^{p}\right)\right)^{3}$ and $\delta^{p} \in L^{\infty}\left(\Gamma_{c}^{p}\right)$. Let us assume that the normal contact stress $T_{\nu} \in L^{\infty}\left(\Gamma_{c}\right), T_{\nu} \leq 0$, is known apriori so that one can evaluate the slip bound $g$ on $\Gamma_{c}$ by $g=F\left(-T_{\nu}\right)$, where $F=F^{p}>0$ is a coefficient of friction on $\Gamma_{c}^{p}$. Denote $g^{p}=\left.g\right|_{\Gamma_{c}^{p}}$.

The primal formulation of the contact problem with given friction reads 


$$
\min \mathcal{J}(\mathbf{v}) \quad \text { subject to } \quad \mathbf{v} \in \mathcal{K},
$$

where

$$
\mathcal{J}(\mathbf{v})=\frac{1}{2} a(\mathbf{v}, \mathbf{v})-b(\mathbf{v})+j(\mathbf{v})
$$

is the total potential energy functional with the bilinear form $a$ representing the inner energy of the bodies and with the linear form $b$ representing the work of the applied forces $\mathbf{t}^{p}$ and $\mathbf{f}^{p}$, respectively. The sublinear functional $j$ represents the work of friction forces

$$
j(\mathbf{v})=\sum_{p=1}^{s} \int_{\Gamma_{c}^{p}} g^{p}\left\|\mathbf{v}_{t}^{p}\right\| d s,
$$

where $\mathbf{v}_{t}^{p}$ is the projection of the displacement $\mathbf{v}^{p}$ on the plane tangential to the unit outer normal vector to $\Gamma_{c}^{p}$ denoted by $\boldsymbol{\nu}^{p} \in\left(L^{\infty}\left(\Gamma_{c}^{p}\right)\right)^{3}$. Let us introduce unit tangential vectors $\mathbf{t}_{1}^{p}, \mathbf{t}_{2}^{p} \in\left(L^{\infty}\left(\Gamma_{c}^{p}\right)\right)^{3}$ such that the triplet $\mathcal{B}=\left\{\boldsymbol{\nu}^{p}(\mathbf{x}), \mathbf{t}_{1}^{p}(\mathbf{x}), \mathbf{t}_{2}^{p}(\mathbf{x})\right\}$ is an orthonormal basis in $\mathbb{R}^{3}$ for almost all $\mathbf{x} \in \Gamma_{c}^{p}$ and denote $v_{t_{1}}^{p}=\mathbf{v}^{p} \cdot \mathbf{t}_{1}^{p}, v_{t_{2}}^{p}=\mathbf{v}^{p} \cdot \mathbf{t}_{2}^{p}$. Then $\mathbf{v}_{t}^{p}=\left(0, v_{t_{1}}^{p}, v_{t_{2}}^{p}\right)$ with respect to the basis $\mathcal{B}$ so that the norm appearing in $j$ reduces to the Euclidean norm in $\mathbb{R}^{2}$. More details about the formulation of contact problems can be found in Hlaváček et al. [1988].

The Lagrangian $\mathcal{L}: V \times \Lambda_{t} \times \Lambda_{n} \longrightarrow \mathbb{R}$ of the problem $(\mathcal{P})$ is defined by

$$
\mathcal{L}\left(\mathbf{v}, \boldsymbol{\mu}_{t}, \boldsymbol{\mu}_{n}\right)=\frac{1}{2} a(\mathbf{v}, \mathbf{v})-b(\mathbf{v})+\sum_{p=1}^{s} \int_{\Gamma_{c}^{p}} \boldsymbol{\mu}_{t}^{p} \cdot \mathbf{v}_{t}^{p} d s+\sum_{p=1}^{s}\left\langle\mu_{n}^{p}, v_{n}^{p}-\delta^{p}\right\rangle_{\Gamma_{c}^{p}},
$$

where

$$
\begin{aligned}
& \Lambda_{t}=\left\{\boldsymbol{\mu}_{t}=\left(\boldsymbol{\mu}_{t}^{1}, \ldots, \boldsymbol{\mu}_{t}^{s}\right) \in \prod_{p=1}^{s}\left(L^{\infty}\left(\Gamma_{c}^{p}\right)\right)^{2}:\left\|\boldsymbol{\mu}_{t}^{p}\right\| \leq g^{p}, \boldsymbol{\mu}_{t}^{p}=\left(\mu_{t_{1}}^{p}, \mu_{t_{2}}^{p}\right)\right\} \\
& \Lambda_{n}=\left\{\boldsymbol{\mu}_{n}=\left(\mu_{n}^{1}, \ldots, \mu_{n}^{s}\right) \in \prod_{p=1}^{s} H^{-1 / 2}\left(\Gamma_{c}^{p}\right): \mu_{n}^{p} \geq 0\right\}
\end{aligned}
$$

and $\langle\cdot, \cdot\rangle_{\Gamma_{c}^{p}}$ denotes the duality pairing between $H^{-1 / 2}\left(\Gamma_{c}^{p}\right)$ and $H^{1 / 2}\left(\Gamma_{c}^{p}\right)$.

The Lagrange multipliers $\boldsymbol{\mu}_{t}, \boldsymbol{\mu}_{n}$ are considered as functionals on the contact parts of the boundaries. While the first one accounts for the nonpenetration condition, the second one removes the non-differentiability of the sublinear functional as

$$
j(\mathbf{v})=\sup _{\boldsymbol{\mu}_{t} \in \Lambda_{t}} \sum_{p=1}^{s} \int_{\Gamma_{c}^{p}} \boldsymbol{\mu}_{t}^{p} \cdot \mathbf{v}_{t}^{p} d s, \quad \mathbf{v} \in V .
$$

Thus the problem $(\mathcal{P})$ can be replaced by the saddle-point problem as

$$
\min _{\mathbf{v} \in \mathcal{K}} \mathcal{J}(\mathbf{v})=\min _{\mathbf{v} \in V} \sup _{\left(\boldsymbol{\mu}_{t}, \boldsymbol{\mu}_{n}\right) \in \Lambda_{t} \times \Lambda_{n}} \mathcal{L}\left(\mathbf{v}, \boldsymbol{\mu}_{t}, \boldsymbol{\mu}_{n}\right) .
$$


By the mixed formulation of the problem $(\mathcal{P})$, we mean a problem of finding a saddle-point of the Lagrangian $\mathcal{L}$ :

$(\mathcal{M})\left\{\begin{array}{l}\text { Find }\left(\mathbf{u}, \boldsymbol{\lambda}_{t}, \boldsymbol{\lambda}_{n}\right) \in V \times \Lambda_{t} \times \Lambda_{n} \text { such that } \\ a(\mathbf{u}, \mathbf{v})=b(\mathbf{v})-\sum_{p=1}^{s} \int_{\Gamma_{c}^{p}} \boldsymbol{\lambda}_{t}^{p} \cdot \mathbf{v}_{t}^{p} d s-\sum_{p=1}^{s}\left\langle\lambda_{n}^{p}, v_{n}^{p}\right\rangle_{\Gamma_{c}^{p}}, \quad \forall \mathbf{v} \in V \\ \sum_{p=1}^{s} \int_{\Gamma_{c}^{p}}\left(\boldsymbol{\lambda}_{t}^{p}-\boldsymbol{\mu}_{t}^{p}\right) \cdot \mathbf{u}_{t}^{p} d s+\sum_{p=1}^{s}\left\langle\lambda_{n}^{p}-\mu_{n}^{p}, u_{n}^{p}-\delta^{p}\right\rangle_{\Gamma_{c}^{p}} \leq 0, \\ \forall\left(\boldsymbol{\mu}_{t}, \boldsymbol{\mu}_{n}\right) \in \Lambda_{t} \times \Lambda_{n} .\end{array}\right.$

It is well-known that there is a unique saddle-point $\left(\mathbf{u}, \boldsymbol{\lambda}_{t}, \boldsymbol{\lambda}_{n}\right)$ and its first component $\mathbf{u}$ solves the problem $(\mathcal{P})$.

Let us point out that the solution $\mathbf{u} \equiv \mathbf{u}(g)$ of $(\mathcal{P})$ depends on a particular choice of $g \in L^{\infty}\left(\Gamma_{c}\right), g \geq 0$. We can define a mapping $\Phi$ which associates with every $g$ the product $F\left(-T_{\nu}(\mathbf{u}(g))\right)$, where $T_{\nu}(\mathbf{u}(g)) \leq 0$ is the normal contact stress related to $\mathbf{u}(g)$. The classical Coulomb's law of friction corresponds to the fixed point of $\Phi$ which is defined by $g=F\left(-T_{\nu}(\mathbf{u}(g))\right)$. To find it, we can use the method of successive approximations which starts from a given $g^{(0)}$ and generates the iterations $g^{(l)}$ by

$$
g^{(l+1)}=\Phi\left(g^{(l)}\right), l=1,2, \ldots
$$

This iterative process converges provided $\Phi$ is contractive, that is guaranteed for sufficiently small $F$ (see Haslinger [1983]).

\section{Discretizations}

We shall discretize the contact problem with given friction by means of one of the following two approximations.

Approximation $I$ is based on the finite element method applied to the primal $\overline{\text { formulation }(\mathcal{P})}$. We divide the bodies $\Omega^{p}$ into tetrahedron finite elements $\mathcal{T}$ with the maximum diameter $h$ and assume that the partitions are regular and consistent with the decompositions of $\partial \Omega^{p}$ into $\Gamma_{u}^{p}, \Gamma_{t}^{p}$ and $\Gamma_{c}^{p}$. Moreover, we restrict ourselves to the geometrical conforming situation where the intersection between the boundaries of any two different bodies $\partial \Omega^{p} \cap \partial \Omega^{q}, p \neq q$, is either empty, a vertex, an entire edge, or an entire face. On the partitions, we introduce the finite element subspace of $V$ by

$$
V_{h}=V_{h}^{1} \times \cdots \times V_{h}^{s}
$$

with 


$$
V_{h}^{p}=\left\{\mathbf{v}^{p} \in\left(C\left(\Omega^{p}\right)\right)^{3}:\left.\mathbf{v}^{p}\right|_{\mathcal{T}} \in\left(P_{1}(\mathcal{T})\right)^{3} \text { for all } \mathcal{T} \subset \Omega^{p}\right\},
$$

where $P_{m}(\mathcal{T})$ denotes the set of all polynomials on $\mathcal{T}$ of degree $\leq m$. Replacing $V$ by $V_{h}$, we can rewrite the approximative primal formulation $(\mathcal{P})$ into an algebraic form. Then we can proceed to the dual formulation introducing the algebraic mixed formulation (analogously to the continuous setting) and eliminating the primal variables (displacements).

Approximation II is based on the mixed finite element method applied to the mixed formulation $(\mathcal{M})$. The space $V$ is approximated by the same $V_{h}$ as in Approximation $I$. In addition, to approximate the sets $\Lambda_{t}$ and $\Lambda_{n}$, we introduce regular partitions of $\Gamma_{c}^{p}$ formed by rectangles $\mathcal{R}$ with the maximum diameter $H$. Let us point out that this partitions are independent on the partitions of $\Omega^{p}$ used for the approximation of $V$. Let us define

$$
\Lambda_{H}^{p}=\left\{\lambda^{p} \in L^{2}\left(\Gamma_{c}^{p}\right):\left.\lambda^{p}\right|_{\mathcal{R}} \in P_{0}(\mathcal{R}) \text { for all } \mathcal{R} \subset \Gamma_{c}^{p}\right\} .
$$

Repleacing $L^{\infty}\left(\Gamma_{c}^{p}\right)$ and $H^{-1 / 2}\left(\Gamma_{c}^{p}\right)$ by $\Lambda_{H}^{p}$ in the definitions of $\Lambda_{t}$ and $\Lambda_{n}$, we obtain their approximations $\Lambda_{t, H}$ and $\Lambda_{n, H}$, respectively. The approximative mixed formulation $(\mathcal{M})$ can be reduced again to the dual formulation eliminating the primal variables. If the partitions of $\Gamma_{c}^{p}$ are defined by restrictions of the partitions of the bodies $\Omega^{p}$ then we obtain a variant of the so called mortar method, see Krause and Wohlmuth [2002].

The dual formulations arising from both Approximation I and Approximation II are represented by the quadratic programming problems of the same type:

$$
\min \Theta(\boldsymbol{\lambda}) \quad \text { s.t. } \quad \boldsymbol{\lambda} \in \boldsymbol{\Lambda} \quad \text { and } \quad \mathbf{R}^{\top}\left(\mathbf{f}-\mathbf{B}^{\top} \boldsymbol{\lambda}\right)=\mathbf{0},
$$

with

$$
\begin{aligned}
\Theta(\boldsymbol{\lambda}) & =\frac{1}{2} \boldsymbol{\lambda}^{\top} \mathbf{B K}^{\dagger} \mathbf{B}^{\top} \boldsymbol{\lambda}-\boldsymbol{\lambda}^{\top}\left(\mathbf{B} \mathbf{K}^{\dagger} \mathbf{f}-\mathbf{c}\right), \\
\boldsymbol{\Lambda} & =\left\{\boldsymbol{\lambda}=\left(\boldsymbol{\lambda}_{t_{1}}^{\top}, \boldsymbol{\lambda}_{t_{2}}^{\top}, \boldsymbol{\lambda}_{n}^{\top}\right)^{\top}:\left\|\left(\left(\boldsymbol{\lambda}_{t_{1}}\right)_{k},\left(\boldsymbol{\lambda}_{t_{2}}\right)_{k}\right)\right\| \leq g_{k}, \boldsymbol{\lambda}_{n} \geq \mathbf{0}\right\}, \\
\mathbf{B} & =\left[\begin{array}{l}
\mathbf{N} \\
\mathbf{T}_{1} \\
\mathbf{T}_{2}
\end{array}\right], \quad \mathbf{c}=\left[\begin{array}{l}
\mathbf{d} \\
\mathbf{0} \\
\mathbf{0}
\end{array}\right] .
\end{aligned}
$$

Here, $\mathbf{K}^{\dagger}$ denotes a generalized inverse to the symmetric positive semidefinite stiffness matrix $\mathbf{K}=\operatorname{diag}\left(\mathbf{K}_{1}, \ldots, \mathbf{K}_{s}\right), \mathbf{R}$ is the full rank matrix whose columns span the kernel of $\mathbf{K}$, the full rank matrices $\mathbf{N}, \mathbf{T}_{1}, \mathbf{T}_{2}$ describe projections of displacements at the nodes lying on $\Gamma_{c}$ to the normal and tangential directions, respectively, $\mathbf{f}$ represents the nodal forces, $\mathbf{d}$ is the vector of distances between the bodies and $g_{k}$ are the values of the slip bound at the contact nodes. The difference between Approximation I and Approximation II consists in the different contents of $\mathbf{B}$ and $\mathbf{c}$. 
The minimized functional in $(\mathcal{D})$ is strictly convex and quadratic but the feasible set contains the non-linear constraints $\left\|\left(\left(\boldsymbol{\lambda}_{t_{1}}\right)_{k},\left(\boldsymbol{\lambda}_{t_{2}}\right)_{k}\right)\right\| \leq g_{k}$. This constraints can be treated by the method described in Haslinger et al. [2003] so that the efficient algorithm based on the augmented Lagrangian (see Dostál et al. [2003]) can be applied.

The advantage of Approximation II is that the number of the dual variables is lower compared with Approximation $I$, i.e. the size of the problem $(\mathcal{D})$ is considerably reduced. This happens if $H / h>1$, i.e. if the partitions of $\Gamma_{c}^{p}$ used for the definitions of $\Lambda_{H}^{p}$ are coarser than the partitions of the bodies $\Omega^{p}$ restricted to $\Gamma_{c}^{p}$. The coarser partitions are related to the satisfaction of the Ladyzhenskaya-Babuška-Brezzi condition that guarantees the existence and the uniquenees of the solution. For our particular choice of the spaces, this condition is satisfied if the ratio $H / h$ is sufficiently large (see Haslinger and Hlaváček [1982]). On the other hand, the ratio $H / h$ should not be too large in order to avoid violation of the non-penetration condition that is satisfied in the weak sense only.

The method of successive approximation (MSA) can be implemented so that the problem $(\mathcal{D})$ is solved to evaluate the mapping $\Phi$. We shall use a more efficient version of this method, in which two outer loops (i.e. the iterative steps of (MSA) and the outer loop of the algorithm for solving $(\mathcal{D})$ ) can be connected in one loop. The resulting algorithm can be viewed as the method of successive approximation with an inexact solving of the auxiliary problems with given friction.

\section{Numerical experiments and conclusions}

Let us consider two bricks $\Omega^{1}$ and $\Omega^{2}$ as in Figure 1 made of an elastic isotropic, homogeneous material characterized by Young modulus $E=$ $21.2 \times 10^{10}$ and Poisson's ratio $\sigma=0.277$ (steel). The brick $\Omega^{1}$ is unilaterally supported by the rigid foundation $\Omega^{0}$. The applied surface tractions are in Figure 1, the volume forces vanish. Both contact interfaces $\Gamma_{c}^{1}=\Omega^{0} \cap \Omega^{1}$ and $\Gamma_{c}^{2}=\Omega^{1} \cap \Omega^{2}$ are partitioned by two meshes as in Figure 2 . The mesh defined by restriction of the partitions of the bodies $\Omega^{1}$ and $\Omega^{2}$ is triangular (dotted) while the mesh used for approximation of the Lagrange multipliers is rectangular (solid). Let us point that the meshes on the interfaces do match for the sake of simple implementation of the model problem. Our method can be applied directly to the problems with nonmathing meshes.

Table 1 compares behaviour of our algorithm for Approximation $I$ and Approxiamtion $I I$ with $H / h=2$, 4 . All computations are carried out with 12150 primal variables while the number of the dual variables $n_{d}$ is different. From the results, we conclude that the performance of the algorithm is not too sensitive to the value of the coefficient of fricrion and the efficiency of our algorithm is comparable to solving of the linear problems. Approximation II reduces the size of the dual problem $n_{d}$ with relatively minor effect on the 


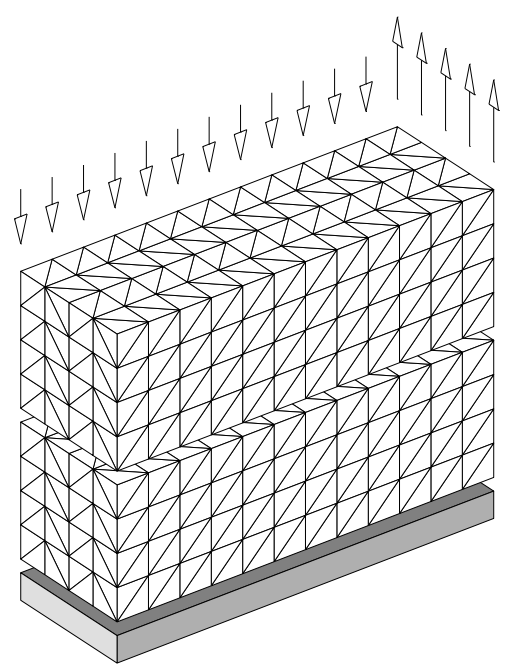

Fig. 1. The model problem with two loaded bodies.

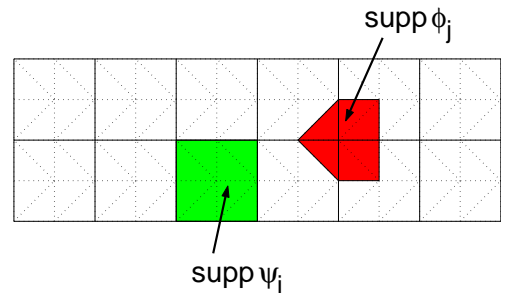

Fig. 2. The meshes on $\Gamma_{c}^{2}$ for $H / h=2$ and the supports of FEM basis functions.

Table 1. Iter denotes the number of outer iterations; $C g$ is the total number of the conjugate gradient steps; err is the relative error.

\begin{tabular}{|c||c|c||c|c|c||c|c|c|}
\hline \multicolumn{1}{|c||}{} & \multicolumn{3}{c||}{ Approx. I } & \multicolumn{6}{c|}{ Approx. II } \\
\hline \hline \multicolumn{1}{|c||}{} & \multicolumn{2}{c||}{$n_{d}=2592$} & \multicolumn{2}{|c|}{$H / h=2, n_{d}=576$} & \multicolumn{2}{|c|}{$H / h=4, n_{d}=144$} \\
\hline \hline$F$ & Iter & $C g$ & Iter & $C g$ & err & Iter & $C g$ & err \\
\hline \hline 0.001 & 24 & 373 & 32 & 299 & 0.0100 & 23 & 147 & 0.0178 \\
\hline 0.01 & 22 & 332 & 26 & 291 & 0.0102 & 16 & 114 & 0.0175 \\
\hline 0.1 & 19 & 331 & 20 & 315 & 0.0120 & 19 & 137 & 0.0147 \\
\hline 1 & 21 & 931 & 21 & 711 & 0.0078 & 24 & 242 & 0.0104 \\
\hline 10 & 16 & 229 & 26 & 213 & 0.0206 & 16 & 117 & 0.0413 \\
\hline
\end{tabular}


solutions compared by $\operatorname{err}=\left\|\mathbf{u}_{I I}-\mathbf{u}_{I}\right\| /\left\|\mathbf{u}_{I}\right\|$, where $\mathbf{u}_{I}$ and $\mathbf{u}_{I I}$ are results of Approximation I and Approximation II, respectively.

Using auxiliary decomposition, results on natural coarse space projections (see Mandel and Tezaur [1996], Klawonn and Widlund [2001]) and quadratic programming (see Dostál and Schöberl [2003], Dostál [2003]), it is possible to show that our algorithm for the problem with given friction is scalable.

\section{References}

Z. Dostál. Inexact semi-monotonic augmented Lagrangians with optimal feasibility convergence for quadratic programming with simple bounds and equality constraints. SIAM J. Num. Anal., 2003. submitted.

Z. Dostál, A. Friedlander, and S. A. Santos. Augmented Lagrangians with adaptive precision control for quadratic programming with simple bounds and equality constraints. SIAM J. Opt., 13:1120-1140, 2003.

Z. Dostál, J. Haslinger, and R. Kučera. Implementation of fixed point method for duality based solution of contact problems with friction. J. Comput. Appl. Math., 140:245-256, 2002.

Z. Dostál and J. Schöberl. Minimizing quadratic functions over non-negative cone with the rate of convergence and finite termination. Comput. Opt. Appl., 2003. submitted.

C. Farhat and F. X. Roux. An unconventional domain decomposition method for an efficient parallel solution of large-scale finite element systems. SIAM J. Sc. Stat. Comput., 13:379-396, 1992.

J. Haslinger. Approximation of the Signorini problem with friction, obeying Coulomb law. Math. Methods Appl. Sci, 5:422-437, 1983.

J. Haslinger, Z. Dostál, and R. Kučera. On splitting type algorithm for the numerical realization of contact problems with Coulomb friction. Comput. Methods Appl. Mech. Eng., 191:2261-2281, 2002.

J. Haslinger and I. Hlaváček. Approximation of the Signorini problem with friction by a mixed finite element method. J. Math. Anal. Appl., 86:99-122, 1982.

J. Haslinger, R. Kučera, and Z. Dostál. An algorithm for the numerical realization of 3D contact problems with Coulomb friction. J. Comput. Appl. Math., 2003. accepted.

I. Hlaváček, J. Haslinger, J. Nečas, and J. Lovíšek. Solution of Variational Inequalities in Mechanics. Springer, Berlin, 1988.

A. Klawonn and O. B. Widlund. FETI and Neumann-Neumann iterative substructuring methods: connections and new results. Communic. Pure Appl. Math., 54:57-90, 2001.

R. Krause and B. Wohlmuth. A Dirichlet-Neumann type algorithm for contact problems with friction. CVS, 5:139-148, 2002.

J. Mandel and R. Tezaur. Convergence of substructuring method with Lagrange multipliers. Numerische Mathematik, 73:473-487, 1996. 